\title{
DESAFIOS PARA AS PESSOAS COM DEFICIÊNCIA VISUAL NO ACESSO À INFORMAÇÃO DIGITAL
}

\section{CHALLENGES FOR THE VISUALLY IMPAIRED IN ACCESSING DIGITAL}

\author{
Karolina Vieira da Silva Bastos ${ }^{a}$ \\ Ivette Kafure Muñoz \\ Patrícia Neves Raposo ${ }^{\circ}$
}

\begin{abstract}
RESUMO
Introdução: Este artigo apresenta um estudo sobre a interação entre a pessoa com deficiência visual e a informação no ambiente digital. Objetivo: Identificar os desafios de acesso à informação enfrentados pelas pessoas com deficiência visual na interação com os ambientes informacionais digitais. Metodologia: Estudo de natureza descritiva, utiliza como abordagem metodológica o método misto - qualitativo e quantitativo -, método de pesquisa levantamento para obter características do grupo estudado, com técnica de entrevista semiestruturada. A amostra da pesquisa é composta por 52 pessoas com deficiência visual, constituindo-se de alunos e funcionários do Centro de Ensino Especial de Deficientes Visuais e usuários e funcionários da Biblioteca Braille Dorina Nowill. Resultados: Os resultados da pesquisa mostram que a inserção dos recursos digitais na vida da pessoa com deficiência visual possibilita ter acesso a mais informações, de maneira rápida e atualizada, com independência e autonomia. Todavia, dificuldades de acesso também são identificadas: dificuldades de acessibilidade nas páginas web, incompatibilidade entre softwares, desconhecimento e inexperiência dos usuários no uso dos recursos digitais e da Internet; e necessidade de conscientizar e encorajar os desenvolvedores a considerarem os usuários com deficiência ao implementar recursos de acessibilidade. Conclusões: Constata-se a importância do acesso à informação digital na vida da pessoa com deficiência visual, entretanto devido às supracitadas barreiras de acesso ainda impostas, demonstra-se a importância de se conhecer as suas especificidades e demandas antes de lhes oferecer tecnologias e serviços para que a informação no ambiente digital se torne cada vez mais acessível e inclusiva.
\end{abstract}

Descritores: Acessibilidade digital. Deficiência visual. Estudo de usuários. Tecnologia

\footnotetext{
a Mestre em Ciência da Informação, Universidade de Brasília (UnB). E-mail: karollinna@gmail.com

b Doutora em Ciência da Informação pela Universidade de Brasília (UnB). Professora na Faculdade de Ciência da Informação na Universidade de Brasília (FCl-UnB). E-mail: ivettekead@gmail.com

c Mestre em Educação pela Universidade de Brasília (UnB). Professora de Educação Inclusiva na Faculdade de Educação da Universidade de Brasília (UnB). E-mail: patricianraposo@gmail.com
} 
Assistiva.

\section{INTRODUÇÃO}

Atualmente, a informação tem se constituído em um bem cuja valorização vem crescendo sob a influência das inovações tecnológicas. As maneiras de disponibilização das informações vêm sendo ampliadas, assim como as formas de interação com essas informações.

Entre os grupos beneficiados pelos avanços das tecnologias, destacam-se as pessoas com deficiência visual, as quais fazem uso muitas vezes, de softwares e hardwares para transpor dificuldades relacionadas à sua limitação visual (ROCHA, 2013). As Tecnologias da Informação e Comunicação (TICs) proporcionam benefícios, autonomia e independência na esfera educacional, profissional, doméstica, de lazer e de entretenimento para todos os seguimentos da sociedade (SONZA, 2008). Mas, apesar das diversas vantagens que tais tecnologias computacionais fazem emergir, elas manifestam também que um usuário pode ter dificuldades de acesso, navegação ou não conseguir compreender as informações veiculadas (ROCHA, 2013).

Garantir a acessibilidade na web não é um processo trivial, devido às distintas soluções requeridas para satisfazer as necessidades de usuários com capacidades diversas. Não é apenas uma tarefa técnica ligada a normas para a construção dos websites e manipulação de artefatos tecnológicos, seja por meio do uso de recursos tecnológicos no estado natural ou pelo uso de Tecnologia Assistiva. Além do conhecimento das tecnologias e suas possibilidades, também é necessário compreender o contexto em que se dá o acesso a estes conteúdos e conhecer conceitos, leis e os diversos tipos de deficiências (ROCHA, 2013).

Diante desse contexto, averiguando a importância de se conhecer as particularidades do usuário com deficiência visual e compreender suas especificidades de acesso antes de thes oferecer tecnologias e serviços, a pesquisa pretendeu responder ao seguinte questionamento: Quais os desafios de acesso à informação enfrentados pelas pessoas com deficiência visual na interação com os ambientes informacionais digitais? Para responder à questão da pesquisa, definiuse como objetivo da pesquisa: identificar os desafios de acesso à informação 
enfrentados pelas pessoas com deficiência visual na interação com os ambientes informacionais digitais. Os objetivos específicos, necessários para alcançar o objetivo geral, são: 1) Identificar o perfil demográfico da pessoa com deficiência visual integrante da amostra da pesquisa; 2) Identificar as necessidades de informação que a pessoa com deficiência visual busca satisfazer ao acessar os ambientes digitais; 3 ) Identificar os recursos digitais de acessibilidade que a pessoa com deficiência visual usa para obter acesso à informação digital; e 4) Identificar as facilidades e as dificuldades encontradas pela pessoa com deficiência visual ao acessar a informação por meio dos recursos digitais.

\section{ACESSIBILIDADE DIGITAL}

A acessibilidade no ambiente digital consiste em remover barreiras que impeçam qualquer pessoa de fazer uso de sistemas computacionais. Ou seja, a acessibilidade digital proporciona informações e serviços em meios digitais que pretende ser isonômica para todas as pessoas, independentemente do tipo de usuário, situação ou ferramenta (KADE et al., 2013). Dentro da acessibilidade digital é possível destacar a acessibilidade na Internet como um dos temas mais estudados e difundidos atualmente (KADE et al., 2013). Isso se dá principalmente porque nos últimos tempos o maior obstáculo enfrentado pelas pessoas com deficiência reside no acesso à informação e, por conseguinte, a aspectos importantes relacionados à informação, como a educação, o trabalho e o lazer. A preocupação atual dos defensores da acessibilidade está em garantir que esses princípios sejam observados também no espaço digital, o espaço da informática e das comunicações (TORRES; MAZZONI; ALVES, 2002).

Nesse sentido, para garantir o crescimento da web, levando ao seu potencial máximo de maneira que os sítios sejam acessíveis a todos, independentemente dos equipamentos, navegadores usados ou de necessidades especiais, esforço internacional notável, pró-acessibilidade no espaço digital, tem sido feito pelo World Wide Web Consortium (W3C), comitê internacional formado por grandes empresas da Internet, que atua como gestor de diretivas para a Internet (TORRES; MAZZONI; ALVES, 2002). Assim, com a finalidade de definir recomendações para a construção de páginas web e a outros documentos disponibilizados no espaço 
digital, o comitê criou o Web Accessibility Initiative (WAI), cuja principal atribuição é elaborar e manter um conjunto de recomendações que, quando seguidas, garantem a construção de sítios com conteúdo acessível para todas as pessoas, independente do hardware que utilizam, software, infraestrutura de rede, idioma, cultura, localização geográfica ou capacidade física e mental (WORLD WIDE WEB CONSORTIUM, 2019b).

Em decorrência desse trabalho, o WAI publicou, em maio 1999, um guia com diretrizes para acessibilidade do conteúdo web, chamado Web Content Accessibility Guidelines (WCAG 1.0). As diretrizes são destinadas para todos os desenvolvedores de conteúdo web e para os desenvolvedores de ferramentas de autoria (WORLD WIDE WEB CONSORTIUM, 1999). Em dezembro de 2008, esse guia foi atualizado, dando origem ao WCAG 2.0, o qual consiste, até o momento, na principal referência mundial em termos de acessibilidade de conteúdo web (WORLD WIDE WEB CONSORTIUM, 2008).

No Brasil, a acessibilidade na Internet começou a trilhar seu caminho com o Decreto 5.296, de 2004 (BRASIL, 2004), que regulamenta a Lei 10.098, de 2000 (BRASIL, 2000), que estabelece normas gerais e critérios básicos para a promoção da acessibilidade das pessoas com deficiência ou com mobilidade reduzida. Tal decreto, em seu artigo 47, torna obrigatória a acessibilidade nos sítios da administração pública, garantindo-lhes o pleno acesso às informações disponíveis. Assim, como o Decreto obriga que as entidades governamentais busquem uma maneira de acessibilizar o conteúdo web de forma padronizada e o mais rápido possível (BRASIL, 2004). Dessa forma, em dezembro de 2005, o Governo Federal, por intermédio do Ministério do Planejamento, Orçamento e Gestão, desenvolveu a cartilha chamada Modelo de Acessibilidade do Governo Eletrônico (eMAG) com a finalidade de ser o norteador no desenvolvimento e a adaptação de conteúdos digitais do Governo Federal, garantindo o acesso a todos. É relevante destacar que o eMAG trata de uma versão especializada do documento do WCAG voltado para - governo brasileiro, porém o eMAG não exclui qualquer boa prática de acessibilidade do WCAG (BRASIL, 2014). A versão atual do modelo, chamada de eMAG 3.1, foi lançada em abril de 2014 e foi desenvolvida por meio da parceria entre o Departamento de Governo Eletrônico, da Secretaria de Logística e 
Tecnologia da Informação do Ministério do Planejamento, Orçamento e Gestão e o Projeto de Acessibilidade Virtual da Secretaria de Educação Profissional e Tecnológica do Ministério da Educação. A versão 3.1 do eMAG apresenta melhorias no conteúdo do texto para torná-lo mais compreensível (BRASIL, 2014). Entretanto, mesmo com todas essas iniciativas, nota-se que as oportunidades de acesso a ambientes digitais informacionais no contexto da pessoa com deficiência visual ainda se distinguem das oportunidades das pessoas que enxergam, pois atualmente a maioria dos sítios e software ainda possuem barreiras de acessibilidade tornando difícil ou impossível para muitas pessoas com deficiência fazer uso da web (WORLD WIDE WEB CONSORTIUM, 2019a). Dessa forma, verifica-se que ainda há caminhos a serem percorridos para que alcance $o$ acesso pleno a informação no ambiente digital.

\section{DEFICIÊNCIA VISUAL E TECNOLOGIA ASSISTIVA}

A deficiência visual, classificada em cegueira e baixa visão, designa uma condição irreversível de perda ou redução da resposta visual em ambos os olhos em caráter permanente, mesmo após tratamento médico, cirurgias ou o uso de lentes (INSTITUTO BENJAMIN CONSTANT, 2005; ROCHA, 2013). Sob essas duas designações encontra-se um vasto número de distúrbios visuais, em pessoas com diversos graus de visão, inclusive residual, e há também diversos termos e definições entre a legislação e outras áreas, como a médica, a de reabilitação e a pedagógica (ROCHA, 2013).

A definição médica é abalizada por duas escalas oftalmológicas que ajudam a estabelecer a existência de grupamentos de deficiências visuais: a acuidade visual (isto é, aquilo que se enxerga a determinada distância) e o campo visual (a amplitude da área alcançada pela visão) (CONDE, 2012). Para avaliar a acuidade visual de uma pessoa, um método universalmente aceito, é a escala optométrica decimal de Snellen, também conhecida como Tabela de Snellen. (ZAPPAROLI; KLEIN; MOREIRA, 2009). A Tabela consiste em um conjunto de símbolos alinhados sobre uma carta branca, com a diminuição de tamanho dos símbolos da linha de cima para a de baixo, numa proporção direta de distância e tamanho baseados em uma escala decimal que varia de 0,1 a 1 , em que a pessoa avaliada visualiza cada 
símbolo, utilizando um olho de cada vez, e informa se está enxergando nitidamente ou não cada símbolo. Conforme o número de linhas visualizadas pela pessoa, é possível avaliar o grau de comprometimento visual e alcançar um diagnóstico (MALHEIROS, 2013).

Para ler a Tabela de Snellen, conforme explicam Polina e Silva (2013), devese observar os números fracionários que aparecem no final de cada linha de letras. Tomando como exemplo 20/40, o primeiro número corresponde à distância em pés entre a Tabela e a pessoa avaliada, em que 20 pés correspondem a 6 metros. Já o segundo número, 40 pés, corresponde à linha de letras; uma pessoa com visão normal enxergaria a 40 pés, porém, alguém com problemas visuais sem correção óptica ou com correção, mas que possui baixa visão, enxerga apenas a 20 pés ou a 5 metros. Assim, o numerador 20 pés permanece fixo, pois é a distância entre a pessoa avaliada e a tabela.

Assim, em uma definição médica uma pessoa é considerada cega quando sua acuidade visual é igual ou inferior a 20/400 (0,05 em escala decimal), ou seja, se ela pode ver a 20 pés (6 metros) o que uma pessoa de visão normal pode ver a 400 pés (120 metros) (WORLD HEALTH ORGANIZATION, 2016), ou se o diâmetro mais largo do seu campo visual subentende um arco não maior de 20 graus, ainda que sua acuidade visual nesse estreito campo possa ser superior a 20/400 (CONDE, 2012). Nesse contexto, caracteriza-se uma pessoa com baixa visão aquela que possui acuidade visual nos valores contidos entre 20/60 (0,3 em escala decimal) e 20/400 (0,05 em escala decimal) (WORLD HEALTH ORGANIZATION, 2016).

A definição médica é bastante utilizada para fins sociais (BRASIL, 2001). Nesse sentido, na legislação a definição médica é utilizada como balizador. Segundo a conceituação prevista no Decreto no 3.298, de 1999 (BRASIL, 1999) modificado pelo Decreto № 5.296, de 2004 (BRASIL, 2004) a deficiência visual classifica-se em cegueira e baixa visão. A cegueira, em escala decimal, corresponde a acuidade visual igual ou menor que 0,05 no melhor olho, com a melhor correção óptica. Já a baixa visão corresponde, em escala decimal, a acuidade visual entre 0,3 e 0,05 no melhor olho, com a melhor correção óptica, e se a somatória do campo visual em ambos os olhos for igual ou menor que $60^{\circ}$; ou a 
ocorrência simultânea de umas das condições anteriores (BRASIL, 2004).

Entretanto, para fins educacionais, como a definição médica não abarca em sua conceituação o potencial visual útil para a execução de tarefas, não deve servir como principal guia para a abordagem educacional (BRASIL, 2001). Assim, pedagogicamente, a visão funcional da pessoa com deficiência visual é a diretriz da conceituação adequada para esse fim, de forma que a avaliação da visão irá considerar a acuidade visual, o campo visual e o uso eficiente do potencial da visão (SÁ; CAMPOS; SILVA, 2007).

Nesse sentido, Kulpa (2009) afirma que a aprendizagem visual não é dependente somente dos olhos, mas também da capacidade do cérebro em captar, codificar, selecionar e organizar imagens percebidas pelos olhos. $O$ ato de ver é uma experiência direta, na qual as imagens transmitem informações e representam a máxima aproximação que se pode ter da realidade. Essas imagens são associadas com outras mensagens sensoriais e armazenadas na memória para serem lembradas mais tarde. Assim, Kulpa (2009) ainda afirma que, o desenvolvimento da eficiência visual necessita de amadurecimento dos fatores ópticos e do uso das funções realizadas pelo cérebro. Nesse aspecto, Sá, Campos e Silva (2007) explicam que a eficiência visual se dá por meio da qualidade e aproveitamento do potencial visual conforme as condições de estimulação e de ativação das funções visuais, o que significa que fatores emocionais, condições ambientais e contingenciais de vida do indivíduo interferem diretamente no uso potencial da visão.

Diante desse contexto, verifica-se a importância de estudar o usuário e compreender as características biológicas, as experiências individuais e a herança sociocultural antes de oferecer qualquer tipo de informação em qualquer tipo de recurso ou suporte.

Outro aspecto a ser observado em relação a essa oferta de informação é que os recursos oferecidos para a interação das pessoas com o ambiente digital são altamente dependentes do sentido da visão, tanto para a leitura e localização de informações quanto para a percepção de conteúdos gráficos bastante usados em interfaces gráficas em geral e em sítios, especificamente; da cognição, para a interpretação e compreensão dos conteúdos e do sistema motor, para uso do 
teclado, do mouse e de telas sensíveis ao toque (ROCHA, 2013). Nesse contexto, verifica-se que a pessoa com deficiência visual em quaisquer desses sistemas possui o acesso à informação bastante limitado, inclusive muitas vezes é até mesmo privado desse acesso, caso utilize os recursos de hardware ou software no estado natural que o mundo digital oferece, devido a recursos utilizados principalmente na forma de representações essencialmente visuais para transmitir informações (ROCHA, 2013; SONZA et al., 2013).

Perante o desafio de atender as necessidades diferenciadas dos usuários com deficiência visual e/ ou com outras deficiências e limitações, iniciativas diversas resultam na construção de Tecnologia Assistiva, normas e leis com vistas a promover a acessibilidade dessas pessoas no ambiente digital. Assim, com o desenvolvimento do campo da Tecnologia Assistiva, esses usuários possuem à sua disposição recursos de hardware (lupa eletrônica, impressora Braille, Braille falado, thermoform, entre outros) e software (LentePro, leitores de tela, Braille Fácil, entre outros) apropriados para mediar e facilitar o seu acesso ao ambiente digital (ROCHA, 2013).

Com a convergência tecnológica e a crescente demanda em estar conectado constantemente, independente do local, popularizaram-se as soluções em comunicação portátil, tendo o smartphone um papel central entre elas (FAÇANHA; VIANA; PEQUENO, 2011).

Os leitores de tela para dispositivos móveis com tela touch screen (tela de toque) possuem a leitura constituída em movimentos/gestos, isto é, tem que possibilitar que pessoas com dificuldades visuais percorram e escolham as opções por meio de toques na tela para ouvir uma descrição do item sob o seu dedo, podendo passar comandos para o seu dispositivo por meio de toques, arrastes ou deslizes (FAÇANHA, 2012).

Cabe ressaltar que a indicação da tecnologia mais apropriada para cada caso depende das características individuais de cada sujeito e da fase de aprendizagem do uso da Tecnologia Assistiva (FAÇANHA, 2012). Também, as influências familiares e externas são consideradas como contribuintes potencialmente importantes para a aquisição de habilidades em Tecnologia Assistiva e acesso (MENG EE; LIIBY, 2011). 
A partir do que foi exposto, constata-se que os dispositivos de acessibilidade são essenciais para a pessoa com deficiência visual, pois permitem o acesso a informação digital, assegurando-lhes independência e autonomia, gerando motivação e produzindo oportunidades para que sejam incluídos no ambiente digital e nas comunidades ali contidas (SILVA, 2010).

\section{ESTUDO DE USUÁRIOS ORIENTADOS À PESSOA COM DEFICIÊNCIA VISUAL}

O estudo de usuários pode ser designado como pesquisas que se fazem para saber o que as pessoas precisam em matéria de informação ou se essas pessoas estão satisfeitas e sendo atendidas adequadamente por seus provedores. Esses estudos compreendem, também, a investigação de como e para que a informação é utilizada pelos usuários, e quais os fatores que afetam tal uso, assim como as formas que essas necessidades são expressas e conhecidas dentro de uma área temática ou pelos que utilizam os produtos e serviços de uma unidade de informação ou sistema de informação (FIGUEIREDO, 1979, 1994).

Os estudos de usuários são norteados por três abordagens: a tradicional, a alternativa e a social. Estudos de abordagem tradicional, de acordo com Cunha, Amaral e Dantas (2015), são predominantemente quantitativos, centrados nos sistemas, anteriores à década de 1980, que buscam mensurar as fontes informacionais mais utilizadas ou o grau de satisfação do usuário com determinado serviço. Em contraste com a abordagem tradicional, surgem no início dos anos 1980, os estudos de abordagem alternativa, a qual se caracteriza por estudos centrados no usuário da informação: estado do conhecimento anômalo (Belkin), construção de sentido (Dervin), valor agregado (Taylor) e construtivismo (Kuhlthau), mediante uma orientação qualitativa (CUNHA; AMARAL; DANTAS, 2015). Ou seja, essa abordagem foca sua compreensão no uso da informação em situações particulares, centrando-se no usuário, examinando o sistema somente como este é visto pelo usuário (DERVIN; NILAN, 1986).

A partir de finais da década de 1990, conforme descreve Araujo (2012), surgem novos estudos e perspectivas desenvolvidas no campo dos estudos de usuários, como as teorias de Choo (2003), Godbold (2006), Wilson (2002), 
Tuominen, Talja e Savolainen (2005). Esses estudos começaram a tentar conciliar a abordagem tradicional e a alternativa, buscando superar as tendências que ora viam o usuário como nulo, totalmente determinado pelo seu pertencimento a um perfil sócio demográfico (como na abordagem tradicional), ora viam os usuários como ser isolado, dotado de critérios únicos (totalmente individuais) para julgar a informação, sentindo e definindo isoladamente na sua mente algo como "necessidade de informação" (tal como na abordagem alternativa). Assim, ainda segundo Araujo (2012) diversos autores buscaram superar esse desafio aliando, por um lado, todo o conhecimento acumulado nas décadas anteriores, no campo dos estudos de usuários, com as questões e problematizações surgidas mais recentemente na teorização sobre o conceito de informação na Ciência da Informação.

Portanto, esses estudos ao adotarem uma nova postura, um novo olhar sobre os sujeitos, buscando compreendê-los, bem como suas ações, indissociáveis de seu contexto histórico e sociocultural apresentam propostas de novos caminhos de pesquisa. Nesse sentido, Araujo (2012) aproximou os avanços recentes no campo de estudos de usuários e de comportamento informacional com a proposta de um "paradigma social" - apresentada por Capurro e compartilhada por autores como Hjorland, Frohmann e Brier - o qual propõe o desenho de uma possível "abordagem interacionista" para o campo. Segundo Araujo (2012, p. 149) "[...] numa perspectiva interacionista, o usuário não é totalmente determinado pelo contexto no insere, nem é totalmente isolado ou alheio a ele; a determinação que o contexto exerce existe, é real, mas não é mecânica nem absoluta, é interpretada e alterada pelo sujeito." Portanto, uma perspectiva interacionista volta-se para a percepção da dimensão reciprocamente referenciada dos fenômenos e dos elementos que o compõem.

A partir da compreensão de cada uma dessas abordagens, observa-se que estudos baseados em cada uma delas se aplicam melhor a distintas situações, o que significa que o surgimento de uma abordagem não invalida a outra e, ainda, que não há a melhor ou pior abordagem, mas sim abordagens mais adequadas a cada contexto a ser investigado. Entretanto, cada vez mais o campo de estudo de usuários atribui importância ao contexto do usuário e, para melhor estudá-lo inserido 
neste contexto, a abordagem social se mostra mais adequada por permitir que sejam considerados fatores históricos, culturais, políticos, sociais e econômicos (ROCHA, 2013).

Nesse sentido, ao versar sobre o usuário com deficiência visual, foco deste artigo, verifica-se que a abordagem social, visão sócio-cognitiva, torna-se a abordagem mais adequada para o contexto a ser investigado, visto que, no processo de ensino e aprendizagem da pessoa com deficiência visual é necessário mais que estimular os demais sentidos além da visão, pois, de acordo com Vygotsky (1997), o desenvolvimento e educação das pessoas com deficiência visual se dará pelo processo de compensação social centrado na capacidade da linguagem de superar as limitações produzidas pela impossibilidade de acesso direto à experiência visual. Dessa forma, Vitorini (2015) afirma que o meio ao qual a pessoa com deficiência visual está inserida é fundamental, pois no ambiente em que ela está inserida ela receberá diversos estímulos, a qual vivenciará experiência de contato com o mundo ao seu redor. Assim, quanto maior o estímulo e mais adequado, maior será o desenvolvimento da pessoa com deficiência e esse desenvolvimento estará ligado à sua interação com o meio ao qual está inserida. De tal forma, também quanto maior o conhecimento sobre o meio, maiores serão as referências para que ela consiga obter e assim ampliar sua compreensão sobre o mundo, o que permite observar a contribuição dos estudos sócio-cognitivos para esse campo. Portanto, é possível observar que a visão sócio-cognitiva auxilia nos estudos que contemplem as pessoas com deficiência visual e também oferecem subsídios para a construção de ambientes informacionais mais inclusivos.

\section{PERCURSOS METODOLÓGICOS}

No que concerne ao propósito da pesquisa, o estudo se caracteriza como de natureza descritiva, pois este estudo descreve e compreende sob uma perspectiva aprofundada as características relevantes do fenômeno pesquisado (PINSONNEAULT; KRAEMER, 1993).

Sob a alegação de conhecimento de concepção filosófica pragmática, a pesquisa concentra a atenção no problema de pesquisa na área das ciências sociais e utiliza abordagens pluralísticas para derivar conhecimento sobre o problema. $\mathrm{Ou}$ 
seja, segundo Creswell (2010), a pesquisa com base na concepção filosófica pragmática utiliza múltiplos métodos, técnicas e os procedimentos que melhor se ajustarem às necessidades e propósitos em estudo.

Assim, tendo como base filosófica o pragmatismo, é utilizado como abordagem metodológica o método misto, o qual coleta e analisa os dados tanto de forma qualitativa quanto quantitativa (CRESWELL, 2010). Deste modo, como estratégia de investigação é utilizada a estratégia incorporada concomitante, pois segundo Creswell (2010), essa estratégia visa o uso de uma única fase de coleta de dados para coletar, ao mesmo tempo, dados qualitativos e quantitativos, pois a combinação dos dados dos dois métodos permite enriquecer a descrição dos participantes da pesquisa.

Quanto ao tipo de pesquisa o estudo é classificado como básica, visto que tem como objetivo gerar novos conhecimentos úteis para o avanço das ciências sociais, envolvendo verdades e interesses universais (KAUARK; MANHÃES; MEDEIROS, 2010).

Em relação ao horizonte temporal da pesquisa, está assume a forma transversal, pois a coleta dos dados ocorre em um só momento, pretendendo descrever e analisar o estado de uma ou várias variáveis em um dado momento (SAMPIERI; COLLADO; LUCIO, 1991).

O método de pesquisa adotado é o levantamento, visto que são realizados enunciados descritivos sobre o grupo pesquisado para descobrir a distribuição de certos traços e atributos que determinam as suas características (BABBIE, 2003). Como técnica desse método é utilizada a entrevista para coletar, de uma forma sistemática, dados sobre as pessoas e as suas preferências, os seus pensamentos e os seus comportamentos (BHATTACHERJEE, 2012). A entrevista é desenvolvida de forma semiestruturada, pois é utilizado um roteiro com perguntas principais, complementadas por questões que surgem durante o momento da entrevista, pois segundo Manzini (1991), verifica-se numa pesquisa a importância de que as informações coletadas sejam fruto de associações feitas pelo entrevistado.

Como instrumentos de pesquisa são utilizados: caneta, papel e gravador de áudio, pois segundo Creswell (2010) os pesquisadores que realizam entrevistas devem utilizar anotações escritas à mão em formulários e gravações de áudio e 
recomenda que, mesmo que a entrevista seja gravada, é adequado que os pesquisadores façam anotações, pois os equipamentos eletrônicos estão sujeitos a falhas.

Em relação à técnica para análise de dados é utilizada na abordagem qualitativa a técnica de análise hermenêutica, a qual consiste num tipo de análise de dados em que os textos ou imagens obtidas na entrevista devem ser interpretados pelo pesquisador considerando a experiência do participante da pesquisa dentro do seu contexto histórico-social (BHATTACHERJEE, 2012). Já na abordagem quantitativa a técnica utilizada é a estatística descritiva, a qual permite que uma série de valores de mesma natureza será sintetizada, organizada e descrita por meio de tabelas, gráficos e medidas descritivas (BABBIE, 2003).

O tipo de estratégia de amostragem utilizada é à estratégia por critério, pelo fato de que todos os participantes selecionados atendem critérios estabelecidos para que se garanta a qualidade da pesquisa (MILES; HUBERMAN, 1994). Assim, são critérios estabelecidos para participar da pesquisa: ter deficiência visual total ou parcial; ser alfabetizado; ter acima de 18 anos; saber acessar recursos que proveem informação digital; aceitar participar da pesquisa de forma livre e gratuita; e aceitar o Termo de Consentimento Livre e Esclarecido.

Para selecionar o segmento da população que fez parte da pesquisa, a abordagem de amostragem adotada é a não probabilística intencional, visto que foram selecionados indivíduos e locais para o estudo que podem intencionalmente informar uma compreensão do problema de pesquisa e o fenômeno central no estudo (CRESWELL, 2014).

Como a pesquisa envolveu a participação de seres humanos, foi necessário para a realização da pesquisa que o projeto fosse registrado e aprovado na Plataforma Brasil, base nacional e unificada de registros de pesquisas envolvendo seres humanos para todo o sistema de Comitê de Ética em Pesquisa (CEP) e Comissão Nacional de Ética em Pesquisa (CONEP). Ao ser registrado na Plataforma Brasil o projeto foi encaminhado e recepcionado pelo Comitê de Ética em Pesquisa do Instituto de Ciências Humanas da Universidade de Brasília (CEP/IH). Avaliado em reunião pelos pareceristas responsáveis foi emitido parecer consubstanciado do CEP com a conclusão de que o projeto de pesquisa estava adequado às exigências da 
Resolução CNS 466/2012, 510/2016 e complementares. O projeto foi aprovado pelo CEP/CHS, isentada a necessidade de apreciação da CONEP.

\section{OBJETO DO ESTUDO}

Como objeto de estudo selecionou-se duas instituições públicas do Distrito Federal que possuem o atendimento especializado de pessoas com deficiência visual: a Biblioteca Braille Dorina Nowill, localizada na CNB 01 - Área Especial Taguatinga Norte, única biblioteca pública especializada no atendimento de pessoas com deficiência visual, conveniada com a Secretaria de Estado de Educação do Distrito Federal (SEEDF); e o Centro de Ensino Especial de Deficientes Visuais (CEEDV), localizada na Avenida L2 Sul, Quadra 612 - Área Especial, também única instituição educacional do Distrito Federal e Entorno especializada no atendimento aos estudantes com deficiência visual e gerida pela SEEDF. Ambas as instituições recebem em seus espaços pessoas com deficiência visual das diversas Regiões Administrativas do Distrito Federal e cidades do Entorno, com as mais diversas variadas características pessoais e sociais. Elas também apresentam uma infraestrutura acessível às informações digitais, na qual computadores podem ser utilizados pelas pessoas com deficiência visual para acessar a informação no meio digital. Tais aspectos tornaram esses ambientes favoráveis para a realização da pesquisa.

A partir dessa seleção, realizou-se o levantamento prévio do número de pessoas com deficiência visual que atendem os critérios estabelecidos para participar da pesquisa em ambas instituições. Do público da Biblioteca Braille 24 pessoas com deficiência visual atendem os critérios da amostra, sendo 22 usuários e 2 funcionários que trabalham na instituição. Do público do CEEDV, 84 pessoas com deficiência visual atendem os critérios da amostra, 78 alunos matriculados na aula de digitação do CEEDV e 5 funcionários. No total 108 pessoas com deficiência visual atendem os critérios para participar da pesquisa.

A coleta de dados no CEEDV ocorreu no período de 8 de maio a 27 de junho de 2017, realizaram-se as entrevistas com 32 pessoas -30 alunos e 2 funcionários. $\mathrm{Na}$ Biblioteca Braille as entrevistas ocorreram no período de 26 de julho a 8 de setembro de 2017, procederam-se as entrevistas com 20 pessoas - 18 usuários e 
com os 2 funcionários. No total, foram realizadas 52 entrevistas. Adler e Adler (2012), ao abordar sobre o número de pessoas necessárias para fazer uma amostra adequada para um projeto de pesquisa qualitativa, sugerem aos investigadores de pós-graduação uma amostra em torno de 30 participantes, pois afirmam que esse tamanho oferece vantagem de ir além de um número muito pequeno de pessoas sem impor as dificuldades de recolher dados sem fim, especialmente quando os investigadores são confrontados com restrições de tempo. Também afirmam que 30 é um bom número redondo, particularmente se entrevistas são complementadas com a observação participante. Dessa forma, do número de 108 entrevistas possíveis de serem realizadas na pesquisa alcançou-se o número de 52 entrevistas, obtendo-se todos os dados necessários para alcançar o objetivo desta pesquisa.

\section{RESULTADOS}

Os resultados obtidos na pesquisa estão elencados a seguir, conforme o objetivo relacionado.

\subsection{Perfil Demográfico}

O objetivo que visa identificar o perfil demográfico da pessoa com deficiência visual (sexo, idade, faixa de renda familiar, nível de escolaridade, causa da perda da visão, nível de inclusão digital, entre outros) foi atingido ao conhecer o público estudado e entender o contexto que estão inseridos com base na visão sóciocognitiva do estudo de usuários, na qual primeiramente deve-se compreender quem são esses usuários e posteriormente levantar outros aspectos e realizar análises. Esse levantamento se tornou fator fundamental para o desenvolvimento da pesquisa, servindo de base e auxílio para análise dos outros objetivos da pesquisa.

\subsection{NECESSIDADES DE INFORMAÇÃO}

O objetivo de identificar as necessidades de informação que a pessoa com deficiência visual busca satisfazer ao acessar os ambientes digitais foi apresentado em forma de depoimento dos entrevistados, sendo identificadas necessidades de informação relacionadas a: 
- o conhecimento (busca de informações sobre atualidades, conteúdo para estudos, cultura, entre outros);

- comunicação (e-mail, redes sociais e aplicativos de mensagens); e

- facilitadores de atividades do cotidiano (acesso a conta de banco, marcação de consultas, compras de passagens e pacotes de turismo, entre outros).

A busca por determinado conteúdo modifica-se conforme as atividades desenvolvidas pelos participantes, seus objetivos pessoais e a situação contextual vivenciada por eles. As necessidades de informação da pessoa com deficiência visual não se diferenciam dos usuários que não tem perda da visão, mas tornamse mais amplas conforme a pessoa com deficiência visual precisa de um atendimento próprio em relação ao acesso à informação, pois o suporte informacional e os recursos digitais utilizados para obter acesso à informação devem ser apropriados, de maneira a não impor ou possuir nenhuma barreira excludente.

\subsection{ReCuRsos Digitais de ACESSIBILIDADE}

O objetivo de identificar os recursos digitais de acessibilidade e os locais que a pessoa com deficiência visual usa para obter acesso à informação digital foi atingido por meio da análise dos depoimentos dos entrevistados.

Os recursos de acessibilidade mencionados pelos entrevistados no uso do computador de mesa, netbook e notebook foram:

- o sistema DOSVOX com seus diversos programas;

- os leitores de tela JAWS, NVDA, Virtual Vision e VoiceOver,

- lupa e alto contraste, as funções de acessibilidade do sistema Microsoft Windows; e

- a lupa eletrônica.

Nos dispositivos digitais móveis smartphone e tablet foram destacados:

- o uso dos leitores de Talkback, VoiceOver e o TALKS;

- a realização de ajustes na configuração do smartphone, seja Android ou iOS, como tamanho da fonte e contraste; 
- o uso de aplicativos diversos, os quais são disponibilizados na loja virtual dos smartphones, direcionados à pessoa com deficiência visual; e

- o uso de assistentes pessoais, como Google Assistente (Android) e Siri (iOS).

\subsection{FACILIDADES E DifiCuLDADES de ACESSO À INFORMAÇÃo DigITAL}

O objetivo de identificar as facilidades e as dificuldades encontradas pela pessoa com deficiência visual ao acessar a informação por meio dos recursos digitais também foi alcançado por meio da análise dos depoimentos dos entrevistados.

Em relação às facilidades foi identificado que a inserção dos recursos digitais na vida dos entrevistados possibilitou:

- ter acesso a mais informações, de maneira rápida e atualizada;

- a independência e autonomia em vários aspectos da vida, como para realização de atividades cotidianas, atividades estudantis e para interação e socialização no ambiente digital;

- a democratização do acesso à informação e ao conhecimento; acesso igualitário a informação; e

- inclusão na vida social.

Por outro lado, dificuldades de acesso à informação digital também foram identificadas, como:

- conteúdos de páginas da web contendo elementos gráficos sem descrição textual e sem etiquetas de marcação - principalmente a representação gráfica de links e CAPTCHA;

- inacessibilidade de menus nas páginas web;

- sobreposição de informações nas páginas web, como a inserção súbita de informações extra ou propagandas, gerando camadas de informações que dificultam o fluxo de leitura do leitor de tela;

- uso de animações e efeitos dinâmicos gerados por linguagem JavaScript; 
- escassez de recursos de acessibilidade voltados para ampliação de fontes e alteração de contraste na página da web;

- documentos em formato PDF, em vários aspectos, também foram citados por nem sempre serem acessíveis;

- incompatibilidade entre softwares, os quais comprometem o acesso efetivo a informação digital por não serem construídos conforme os padrões de interação com as páginas web;

- desconhecimento e inexperiência dos usuários tanto no uso dos recursos digitais quanto no uso da Internet, o que traz impacto para que ele em consiga acessar a informação desejada; e

- necessidade de conscientizar e encorajar os desenvolvedores para agregar recursos de acessibilidade que envolvam e considerem as necessidades dos usuários com deficiência.

\section{CONSIDERAÇÕES FINAIS}

O objetivo geral da pesquisa foi identificar os desafios de acesso à informação enfrentados pelas pessoas com deficiência visual na interação com os ambientes informacionais digitais. Para se chegar a este objetivo, foi necessário conhecer os usuários, identificar suas necessidades informacionais, identificar os recursos digitais de acessibilidade, e identificar as facilidades e as dificuldades encontradas ao acessar a informação por meio dos recursos digitais.

Após todo o processo da pesquisa, é possível afirmar que a pessoa com deficiência visual tem se beneficiado efetivamente pelos avanços das TICs, por meio do uso de hardwares e softwares que auxiliam a transpor dificuldades relacionadas ao acesso à informação e também participando ativamente de interações com outros sujeitos e com as informações disponibilizadas na sociedade. Entretanto, a pessoa com deficiência visual ainda encontra limitações ao interagir com os ambientes digitais e um dos caminhos primordiais a se trilhar para garantir a acessibilidade de conteúdos digitais é entender o usuário que faz uso dos recursos tecnológicos. É preciso ampliar o conhecimento em relação as suas necessidades e expectativas e saber se as informações que ele busca no ambiente 
digital estão acessíveis, isto é, se contemplam os requisitos necessários de acessibilidade. Porém, "muitas vezes a acessibilidade é feita, mas sob os olhos de quem enxerga, esquecendo o ponto de vista de quem vai usá-la. Questões como o desenho universal, a usabilidade e a acessibilidade ainda precisam ser discutidas" (MALHEIROS, 2013, p. 262). "Conhecer os usuários, suas demandas auxilia na melhora dos serviços de informações disponíveis" (CASELLI, 2007, p. 87).

Nesse sentido, a pesquisa buscou conhecer o usuário com deficiência visual, saber sobre as suas necessidades e expectativas frente ao uso do ambiente digital e saber se as informações estão acessíveis a fim de ressaltar, por meio da sua percepção, o que ainda pode ser melhorado quanto à acessibilidade da informação no ambiente digital, sendo esta a principal contribuição da pesquisa.

\section{REFERÊNCIAS}

ADLER, Patricia A.; ADLER, Peter. The epistemology of numbers. In: BAKER, Sarah Elsie; EDWARDS, Rosalind. How many qualitative interviews is enough? Expert voices and early career reflections on sampling and cases in qualitative research. Discussion Paper. NCRM. 2012. p. 8-11. Disponível em: http://eprints.ncrm.ac.uk/2273/4/how_many_interviews.pdf. Acesso em: 23 dez. 2019.

ARAUJO, Carlos Alberto Ávila. Paradigma social nos estudos de usuários da informação: abordagem interacionista. Informação \& Sociedade: Estudos, João Pessoa, v. 22, n. 1, p. 145-159, jan./abr. 2012.

BABBIE, Earl. Métodos de pesquisas de survey. Belo Horizonte: UFMG, 2003.

BHATTACHERJEE, A. Social science research: principles, methods, and practices. USF Tampa Bay Open Access Textbooks Collection. Book 3. 2012.

BRASIL. Decreto no 3.298, 20 de dezembro de 1999. Diário Oficial [da] República Federativa do Brasil, Poder Executivo, Brasília, DF, 21 dez. 1999. Seção 1, p. 66. Disponível em: http://pesquisa.in.gov.br/imprensa/jsp/visualiza/index.jsp?data=21/12/1999\&jornal= 1\&pagina=66\&totalArquivos=111. Acesso em: $23 \mathrm{dez} .2019$.

BRASIL. Decreto ํㅜ 5.296, de 2 de dezembro de 2004. Diário Oficial [da] República Federativa do Brasil, Poder Executivo, Brasília, DF, 3 dez. 2004. Seção 1, p. 5. Disponível em: http://pesquisa.in.gov.br/imprensa/jsp/visualiza/index.jsp?data=03/12/2004\&jornal= 1\&pagina=5\&totalArquivos=192. Acesso em: 20 dez. 2019. 
BRASIL. Departamento de Governo Eletrônico. eMAG: Modelo de Acessibilidade em Governo Eletrônico. Versão 3.1. abr. 2014. Disponível em: http://emag.governoeletronico.gov.br/. Acesso em: 19 dez. 2019.

BRASIL. Lei no 10.098, de 19 de dezembro de 2000. Diário Oficial [da] República Federativa do Brasil, Poder Executivo, Brasília, DF, ano 138, n. 244E, 20 dez. 2000. Seção 1, p. 2. Disponível em: http://pesquisa.in.gov.br/imprensa/jsp/visualiza/index.jsp?data=20/12/2000\&jornal= 1\&pagina=2\&totalArquivos=304. Acesso em: 19 dez. 2019.

BRASIL. Ministério da Educação. Secretaria de Educação Especial. Programa de Capacitação de Recursos Humanos do Ensino Fundamental: deficiência visual. Brasília: Ministério da Educação, Secretaria de Educação Especial, 2001. v. 1 fascículos I - II - III. Disponível em:

http://portal.mec.gov.br/seesp/arquivos/pdf/def_visual_1.pdf. Acesso em: 12 dez. 2019.

CASELLI, Brígida Carla Almeida. Acesso à informação digital por portadores de necessidades especiais visuais: estudo de caso do Telecentro Acessível de Taguatinga. 2007. 94 f. Dissertação (Mestrado em Ciência da Informação) Departamento de Ciência da Informação e Documentação, Faculdade de Economia, Administração, Contabilidade e Ciência da Informação e Documentação, Universidade de Brasília, Brasília, 2007.

$\mathrm{CHOO}$, Chun Wei. A organização do conhecimento: como as organizações usam a informação para criar conhecimento, construir conhecimento e tomar decisões. São Paulo: SENAC, 2003.

CONDE, Antônio João Menescal. Definindo a cegueira e a visão subnormal. 2012. Disponível em: http://www.ibc.gov.br/?itemid=94. Acesso em: 11 dez. 2019.

CRESWELL, John W. Investigação qualitativa e projeto de pesquisa: escolhendo entre cinco abordagens. 3. ed. Porto Alegre: Penso, 2014.

CRESWELL, John W. Projeto de pesquisa: métodos qualitativo, quantitativo e misto. 3. ed. Porto Alegre: Artmed, 2010.

CUNHA, Murilo Bastos da; AMARAL, Sueli Angeliza do; DANTAS, Edmundo Brandão. Manual de estudo de usuários da informação. São Paulo: Atlas, 2015.

DERVIN, Brenda; NILAN, Michael. Information needs and uses. In: WILLIAMS, Martha (ed). Annual Review of Information Science and Technology, White Plains, NY, v. 21. p. 3-33, 1986. Disponível em:

https://comminfo.rutgers.edu/ tefko/Courses/612/Articles/zennezdervinnilan86arist .pd. Acesso em: 19 dez. 2019.

FAÇANHA, Agebson Rocha. Uma proposta para acessibilidade visual e táctil 
em dispositivos touchscreen. 2012. 109 f. Dissertação (Mestrado em Ciência da Computação) - Departamento de Computação, da Universidade Federal do Ceará, Fortaleza, 2012.

FAÇANHA, Agebson Rocha; VIANA, Windson; PEQUENO, Mauro Cavalcante. Estudo de interfaces acessíveis para usuários com deficiência visual em dispositivos móveis touchscreen. Nuevas Ideas en Informática Educativa, Santiago de Chile, v. 7, p. 144-149, 2011. Disponível em: http://www.tise.cl/volumen7/TISE2011/Documento18.pdf. Acesso em: 20 dez. 2019.

FIGUEIREDO, Nice Menezes de. Avaliação de coleções e estudo de usuários. Brasília: Associação dos Bibliotecários do Distrito Federal, 1979.

FIGUEIREDO, Nice Menezes de. Estudos de uso e usuários da informação. Brasília: IBICT, 1994.

GODBOLD, Natalya. Beyond information seeking: towards a general model of information behaviour. Information Research, v. 11, n. 4, jul. 2006. Disponível em: http://www.informationr.net/ir/11-4/paper269.html. Acesso em: 14 dez. 2019.

INSTITUTO BENJAMIN CONSTANT. Os conceitos de deficiência: as diversas definições. 2005. Disponível em: http://www.ibc.gov.br/?catid=83\&itemid=396. Acesso em: 12 dez. 2019.

KADE, Adrovane; NASCIMENTO, Gleison Samuel do; SALTON, Bruna Poletto; POTAPCZUK, Diego de Oliveira; SOARES, Fernando Sebenello; ALMEIDA, Jucélia Poletto; NERVIS, Lael; MORO, Ricardo. Acessibilidade virtual. In: SONZA, Andréa Poletto; KADE, Adrovane; FAÇANHA, Agebson; REZENDE, André Luiz Andrade, NASCIMENTO, Gleison Samuel do; ROSITO, Maurício Covolan; BORTOLINI, Sirlei; FERNANDES, Woquiton Lima (orgs.).

Acessibilidade e tecnologia assistiva: pensando a inclusão sociodigital de PNEs. Bento Gonçalves, RS: Instituto Federal do Rio Grande do Sul Campus Bento Gonçalves, 2013. cap. 5, p. 313-364. (Série Novos Autores da Educação Profissional e Tecnológica). Disponível em: http://forumeja.org.br/sites/forumeja.org.br/files/acessibilidade-tecnologiaassistiva\%20(texto\%20complementar).pdf. Acesso em: 20 dez. 2019.

KAUARK, Fabiana da Silva; MANHÃES, Fernanda Castro; MEDEIROS, Carlos Henrique. Metodologia da pesquisa: um guia prático. Itabuna: Via Litterarum, 2010.

KULPA, Cínthia Costa. A contribuição de uma modelo de cores na usabilidade das interfaces computacionais para usuários de baixa visão. 2009. 191 f. Dissertação (Mestrado em Design) - Universidade Federal do Rio Grande do Sul, Porto Alegre, 2009.

MALHEIROS, Tania Milca de Carvalho. Necessidade de informação do usuário 
com deficiência visual: um estudo de caso da biblioteca digital e sonora da Universidade de Brasília. 2013. 305 f. Dissertação (Mestrado em Ciência da Informação) - Universidade de Brasília, Brasília, 2013.

MANZINI, Eduardo J. A entrevista na pesquisa social. Didática, São Paulo, v. 26/27, p. 149-158, 1991.

MENG EE, Wong; LIIBY, Cohen. School, family and other influences on assistive technology use: access and challenges for students with visual impairment in Singapore. British Journal of Visual Impairment, v. 29, n. 2, p. 130-144, may 2011.

MILES, Matthew B.; HUBERMAN, A. Michael. Qualitative data analysis: a sourcebook of new methods. 2nd. ed. Thousand Oaks: Sage, 1994.

PINSONNEAULT, Alain; KRAEMER, Kenneth L. Survey research methodology in management information systems: an assessment. Journal of Management Information Systems, v. 10, n. 2, p. 75-105, sep. 1993.

POLINA, Lidia; SILVA, Vera Lucia Ruiz Rodrigues da. Avaliação da acuidade visual em alunos do ensino fundamental e suas implicações pedagógicas. In: PARANÁ. Governo do Estado. Secretaria de Educação. Os desafios da escola pública paranaense na perspectiva do professor PDE. Cadernos PDE, 2013.

ROCHA, Janicy Aparecida Pereira. (In)acessibilidade na web para pessoa com deficiência visual: um estudo de usuários à luz da cognição situada. 2013. 157 f. Dissertação (Mestrado em Ciência da Informação) - Programa de PósGraduação em Ciência da Informação, da Escola de Ciência da Informaç̧ão, da Universidade Ferderal de Minas Gerais, Belo Horizonte, 2013.

SÁ, Elizabeth Dias de; CAMPOS, Izilda Maria de; SILVA, Myriam Beatriz Campolina. Inclusão escolar de alunos cegos e com baixa visão. In: SÁ, Elizabeth Dias de; CAMPOS, Izilda Maria de; SILVA, Myriam Beatriz Campolina. Formação continuada a distância de professores para $o$ atendimento educacional especializado: deficiência visual. Brasília: SEEP/ SEED/ MEC, 2007. p. 13-38. Disponível em: http://portal.mec.gov.br/seesp/arquivos/pdf/aee_dv.pdf. Acesso em: 23 dez. 2019.

SAMPIERI, Roberto Hernández; COLLADO, Carlos Fernández; LUCIO, Pilar Baptista. Metodología da la investigación. México: McGraw-Hill, 1991.

SILVA, Karolina Vieira da. A inclusão digital e as dificuldades do acesso à informação para pessoas com deficiência visual. 2010. 158 f. Monografia (Bacharel em Biblioteconomia) - Universidade de Brasília, Brasília, 2010.

SONZA, Andréa Poletto; FAÇANHA, Agebson; FÉO, Fabíola; PAGANI, Josiane; GATTO, Juliano; MACHADO, Marco André Santos; MAIA, Nádia; CAINELLI, Rodrigo; FERNANDES, Woquiton Lima. Tecnologia assistiva e software 
educativo. In: SONZA, Andréa Poletto; KADE, Adrovane; FAÇANHA, Agebson; REZENDE, André Luiz Andrade, NASCIMENTO, Gleison Samuel do; ROSITO, Maurício Covolan; BORTOLINI, Sirlei; FERNANDES, Woquiton Lima (orgs.). Acessibilidade e tecnologia assistiva: pensando a inclusão sociodigital de pessoas com necessidades especiais. Bento Gonçalves, RS: Instituto Federal do Rio Grande do Sul Campus Bento Gonçalves, 2013. cap. 4, p. 199-312. (Série Novos Autores da Educação Profissional e Tecnológica). Disponível em: https://drive.google.com/file/d/1wtpwN4govndQFhGOYwtHnCVZ3bCegrJ0/view. Acesso em: 19 dez. 2019.

SONZA, Andréa Poletto. Ambientes virtuais acessíveis sob a perspectiva de usuários com limitação visual. 2008. 313 f. Tese (Doutorado em Informática na Educação) - Programa de Pós-Graduação em Informática na Educação, Centro Interdisciplinar de Novas Tecnologias na Educação, Universidade Federal do Rio Grande do Sul, Porto Alegre, 2008.

TORRES, Elisabeth Fátima; MAZZONI, Alberto Angel; ALVES, João Bosco da Mota. A acessibilidade à informação no espaço digital. Ciência da Informação, Brasília, v. 31, n. 3, p. 83-91, set./dez. 2002. Disponível em: http://www.scielo.br/pdf/ci/v31n3/a09v31n3.pdf. Acesso em: 20 dez. 2019.

TUOMINEN, Kimmo; TALJA, Sanna; SAVOLAINEN, Reijo. The social constructionist viewpoint on information practices. In: FISCHER, Karen E.; ERDELEZ, Sandra; McKECHNIE, Lynne (org.). Theories of information behaviour. Medford: Information Today, 2005. Disponível em: http://migre.me/vjg4a. Acesso em: 22 dez. 2019.

VITORINI, Érica Fernanda. Uso da linguagem documentária na busca da informação em bibliotecas universitárias: a perspectiva dos deficientes visuais. Dissertação (Mestrado em Ciência da Informação) - Faculdade de Filosofia e Ciências, Universidade Estadual Paulista, Marília, 2015.

VYGOTSKY, L. S. Obras escogidas V: fundamentos de defectologia. Madrid: Visor, 1997.

WILSON, Thomas. Alfred Schutz, phenomenology and research methodology for information behaviour research. In: INTERNATIONAL CONFERENCE ON INFORMATION SEEKING IN CONTEXT, 4., 2002. Anais [...]. Lisboa: Universidade Lusíada, 2002.

WORLD HEALTH ORGANIZATION. International Statistical Classification of Diseases and Related Health Problems. 10th rev. Version 2016. Disponível em: http://apps.who.int/classifications/icd10/browse/2016/en\#/H54. Acesso em: 20 dez. 2019.

WORLD WIDE WEB CONSORTIUM. Introduction to web accessibility. 2019a. Disponível em: https://www.w3.org/WAl/intro/accessibility.php. Acesso em: 14 ago. 2018. 
WORLD WIDE WEB CONSORTIUM. Web Accessibility Initiative (WAI). 2019b.

Disponível em: https://www.w3.org/WAl/. Acesso em: 19 dez. 2019.

WORLD WIDE WEB CONSORTIUM. Web Content Accessibility Guidelines (WCAG) 2.0. 2008. Disponível em: https://www.w3.org/TR/WCAG20/. Acesso em: 20 dez. 2019.

WORLD WIDE WEB CONSORTIUM. Web Content Accessibility Guidelines 1.0. 1999. Disponível em: https://www.w3.org/TR/WCAG10/. Acesso em: 14 dez. 2019.

ZAPPAROLI, Márcio; KLEIN, Fernando; MOREIRA, Hamilton. Avaliação da acuidade visual Snellen. Arquivo Brasileiro de Oftalmologia. São Paulo, v. 72, n. 6, 2009. Disponível em: http://www.scielo.br/pdf/abo/v72n6/08.pdf. Acesso em: 22 dez. 2019.

\title{
CHALLENGES FOR THE VISUALLY IMPAIRED IN ACCESSING DIGITAL INFORMATION
}

\begin{abstract}
Introduction: This paper presents a study on the interaction between a visually impaired person and information in the digital environment. Objective: Identify the challenges of information access faced by people with visual impairment when interacting with digital information environments. Methodology: A descriptive study, using the mixed method qualitative and quantitative - as methodological approach, through the research method survey, with semi-structured interview technique to obtain characteristics of the group studied. The research sample is composed of 52 people with visual impairment, consisting of students and staff of the Special Education Center for the Visually Impaired and users and staff of the Braille Library Dorina Nowill. Results: The results of the research show that the insertion of digital resources in the life of the visually impaired person allows access to more information, in a fast and updated way, with independence and autonomy. However, access difficulties are also identified: accessibility difficulties in web pages, incompatibility between softwares, the unfamiliarity and inexperience of users in the use of digital resources and the Internet; and the need to educate and encourage developers to consider impairment users when implementing accessibility features. Conclusions: Was confirmed the importance of accessing digital information in the life of the visually impaired person, however, due to the above-mentioned barriers of access still imposed, demonstrates the importance of knowing their specificities and demands before offering them technologies and services so that information in the digital environment could become increasingly accessible and inclusive.
\end{abstract}

Descriptors: Assistive Technology. Digital accessibility. Users study. Visual impairment.

\section{DESAFÍOS PARA LOS DISCAPACITADOS VISUALES EN EL ACCESO A LA INFORMACIÓN DIGITAL}




\section{RESUMEN}

Introducción: Este artículo presenta un estudio sobre la interacción entre una persona con discapacidad visual y la información en el entorno digital. Objetivo: Identificar los desafíos de acceso a la información que enfrentan las personas con discapacidad visual al interactuar con entornos de información digital. Metodología: Estudio descriptivo, utilizando el método mixto, cualitativo y cuantitativo, como enfoque metodológico, por medio de la encuesta de métodos de investigación, con técnica de entrevista semiestructurada, para obtener características del grupo estudiado. La muestra de investigación está compuesta por 52 personas con discapacidad visual, compuesta por estudiantes y personal del Centro de educación especial para personas con discapacidad visual y usuarios y personal de la Biblioteca Braille Dorina Nowill. Resultados: Los resultados de la investigación muestran que la inserción de recursos digitales en la vida de la persona con discapacidad visual permite el acceso a más información, de manera rápida y actualizada, con independencia y autonomía. Sin embargo, también se identifican dificultades de acceso: dificultades de accesibilidad en páginas web, incompatibilidad entre softwares, desconocimiento e inexperiencia de los usuarios en el uso de recursos digitales e Internet; y la necesidad de educar y alentar a los desarrolladores a considerar a los usuarios discapacitados al implementar las funciones de accesibilidad. Conclusiones: Se confirma la importancia de acceder a la información digital en la vida de la persona con discapacidad visual, sin embargo, debido a las barreras de acceso que aún se imponen anteriormente mencionadas, demuestra la importancia de conocer sus especificidades y demandas antes de ofrecerles tecnologías y servicios para que la información en el entorno digital sea cada vez más accesible e inclusiva.

Descriptores: Accesibilidad digital. Discapacidad visual. Estudio de usuarios. Tecnología Asistencial.

Recebido em: 23.12 .2019

Aceito em: 10.06.2020 